\section{ASPP2 is a haploinsufficient tumor suppressor that cooperates with p53 to suppress tumor growth}

\author{
Virginie Vives, ${ }^{1,3}$ Jian Su, ${ }^{1,3}$ Shan Zhong, ${ }^{1,3}$ \\ Indrika Ratnayaka, ${ }^{1}$ Elizabeth Slee, ${ }^{1}$ \\ Robert Goldin, ${ }^{2}$ and Xin $\mathrm{Lu}^{1,4}$ \\ ${ }^{1}$ Ludwig Institute for Cancer Research, University College \\ London, London W1W 7BS, United Kingdom; ${ }^{2}$ Department of \\ Pathology, Imperial College London, Faculty of Medicine at \\ St. Mary's, London W2 1PG, United Kingdom
}

ASPP2 stimulates the apoptotic function of the p53 family in vivo. We show here that $A S P P 2^{-/-}$pups died before weaning. This postnatal lethality was significantly enhanced in $p 53^{+/-}$background and both deletions are synthetic lethal. ASPP2 ${ }^{+/-}$mice developed spontaneous tumors. The tumor onset was accelerated by $\gamma$-irradiation or in $\mathrm{p}^{+3^{+/-}}$background. Tumors derived from $A S P P 2^{+/-}$ mice retained wild-type $A S P P 2$ allele even though some of them lost $p 53$. These provide the first genetic evidence that ASPP2 is a haploinsufficient tumor suppressor that shares overlapping function(s) with p53 in mouse development and tumor suppression.

Received November 25, 2005; revised version accepted February 27, 2006.

ASPP2 belongs to the evolutionarily conserved ASPP (ankyrin repeats, $\mathrm{SH} 3$ domain, and proline-rich region containing protein) family of proteins (Samuels-Lev et al. 2001; Bergamaschi et al. 2003; Slee and Lu, 2003). ASPP2 was previously identified as an interacting partner for a number of proteins, including p53 (Iwabuchi et al. 1994), Bcl-2 (Naumovski and Cleary 1996), RelA p65 (Yang et al. 1999), Yap (Espanel and Sudol 2001), APC-2(APCL) (Nakagawa et al. 2000), APP-BP1 (Chen et al. 2003), and protein phosphatase 1 (PP1) (Helps et al. 1995). All of these proteins, with the exception of APP-BP1, interact with the C-terminal region of ASPP2. The residues involved in the interactions between ASPP2 and p53 or PP1 were identified through crystal structure studies (Gorina and Pavletich 1996; Egloff et al. 1997).

ASPP2 binds to the evolutionarily conserved DNAbinding domain of p53, and all ASPP2 contact residues of p53 are mutated in human cancers (Gorina and Pavletich 1996). This suggests that ASPP2/p53 interaction might have a tumor suppression function. Consistent with this, the ASPP2/p53 interaction specifically stimulates the apoptotic function of p53 (Samuels-Lev et al. 2001)

[Keywords: ASPP2; knockout mice; tumor suppressor; p53; rhabdomyosarcoma]

${ }^{3}$ These authors contributed equally to this work.

${ }^{4}$ Corresponding author.

E-MAIL x.lu@ludwig.ucl.ac.uk; FAX 44-20-7878-4040.

Article and publication are at http://www.genesdev.org/cgi/doi/10.1101/ gad.374006. and its family members p63 and p73 (Bergamaschi et al. 2004). This is achieved through the ability of ASPP2 to enhance the DNA-binding and transactivation function of p53, p63, and p73 on promoters of $B a x$, PIG3, and PUMA, but not on $p 21^{\text {waf } 1}$ nor on $m d m 2$.

The biological significance of the interaction between ASPP2 and non-p53 family proteins remains unclear. ASPP2 does not bind to the catalytic domain of PP1, although the peptide of ASPP2 containing the PP1-binding motif (RXVF) is able to inhibit the phosphatase activity of PP1 (Egloff et al. 1997). The binding of ASPP2 to APP-BP1 prevents the apoptotic function of APP-BP1 in neuronal cells by influencing the neddylation of cullin, suggesting that ASPP2 might have an antiapoptotic function in some contexts (Chen et al. 2003). The ASPP2/p65 interaction did not have a dramatic effect on the transactivation function of p65 (Yang et al. 1999).

In order to provide an in vivo model system to study the biological functions of ASPP2, we generated and characterized ASPP2-deficient mice. This revealed that ASPP2 is required during the development of the brain and heart. Also, heterozygosity of ASPP2-null allele significantly accelerated tumor development. To test the biological importance of the ASPP2/p53 interaction, we examined the kinetics, frequency, and spectrum of tumors that occurred spontaneously in compound mice deficient for both ASPP2 and p53.

\section{Results and Discussion}

\section{ASPP2-deficient mice exhibit postnatal lethality around weaning}

To study the functional importance of ASPP2 in vivo, we inactivated the murine ASPP2 gene in IT2 embryonic stem (ES) cells using a targeting vector in which exon 3 of the mouse ASPP2 gene had been deleted to destroy the ORF of ASPP2 (Fig. 1A). Heterozygous ES clones were injected into C57BL/6J blastocysts, and this gave rise to germline transmitting chimeric mice that were crossed onto the $\mathrm{C} 57 \mathrm{BL} / 6 \mathrm{~J}$ background. The resulting heterozygous mice (Fig. 1B) appeared normal and fertile, and were used to breed ASPP 2 homozygous mutant progeny. Deletion of the exon 3 sequence of the ASPP2 gene was confirmed by the absence of the wild-type 368-base-pair (bp) RT-PCR product and detection of a deleted 268-bp ASPP2 RT-PCR product (Fig. 1C). Deletion of the ASPP2 protein was confirmed in null embryos using two different polyclonal antibodies raised, respectively, against protein fragments from the N-terminal (residues 59-383) (Fig. 1D) or the middle (residues 500-600) part of ASPP2 (data not shown). A reduced expression level of ASPP2 protein $(50 \%)$ was observed in heterozygotes, compared with wild-type littermates (Fig. 1D).

Of 1065 postnatal offspring (postnatal day 5 [P5] to P30) derived from heterozygous matings, only 68 (6.4\%) were homozygous for the ASPP2-null allele. The male:female ratio of surviving homozygotes was 1:1.08 ( $n=23$ mice sexed). Hence, the postnatal survival of ASPP2-null pups was far below the expected frequency of $25 \%$, but sex-independent. A timed-mating study revealed that the expected percentage of ASPP2-null embryos survived up to embryonic day 18.5 (E18.5), suggest- 


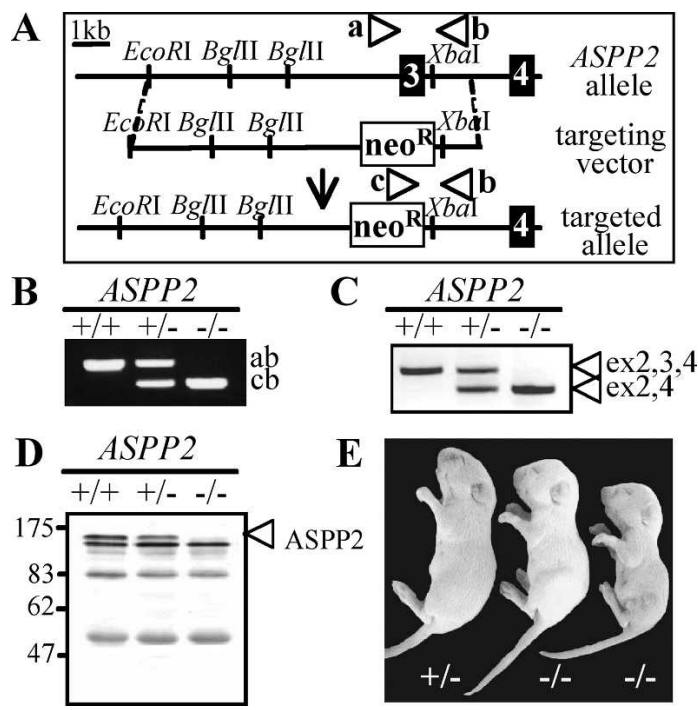

Figure 1. Targeted disruption of the ASPP2 gene. (A) Schematic representation of part of wild-type ASPP2 (top), targeting vector (middle), and targeted ASPP2 allele (bottom). Black boxes represent ASPP2 exons. In the targeting vector, exon 3 is replaced by a neomycin-resistance gene cassette (neo). The location of the primers a, $\mathrm{b}$, and $\mathrm{c}$ used for genotyping (see $B)$ is indicated. $(B)$ PCR genotyping from tail DNA of $A S P P 2^{+/+}, A S P P 2^{+/-}$, and $A S P P 2^{-/-}$mice using specific primers $\mathrm{a}, \mathrm{b}$, and $\mathrm{c}$. Note the $1.1-\mathrm{kb}$ wild-type and $0.9-\mathrm{kb}$ mutant PCR products. $(C)$ RT-PCR of RNA extracted from E13.5 brains of $A S P P 2^{+/+}, A S P P 2^{+/-}$, and $A S P P 2^{-/-}$embryos using specific primers located in exons (ex) 2 and 4 . (D) Western blot analysis of ASPP2 protein, using the S32 antibody, in E13.5 brains of $A S P P 2^{+/+}$, $A S P P 2^{+/-}$, and $A S P P 2^{-/-}$embryos. (E) Phenotype of P9 pups with indicated ASPP2 genotypes.

ing that most of them died around birth (data not shown). All ASPP2-heterozygous mice developed normally, whereas ASPP2-null pups that survived birth were runty with a domed head shape (Fig. 1E). The majority died between 6 and $30 \mathrm{~d}$ of age, with the remainder dying shortly after weaning. Histological examination revealed that the postnatal death of ASPP2-null pups was likely due to a combination of hydrocephalus and heart abnormalities (data not shown).

Previous studies demonstrated that ASPP1 and ASPP2 are activators of the p53 family members (Samuels-Lev et al. 2001; Bergamaschi et al. 2004) and their amino acid sequences are highly homologous. Both proteins exhibit similar biological functions in all the assays performed so far (Samuels-Lev et al. 2001; Bergamaschi et al. 2004). The fact that the ASPP2-null pups exhibit severe developmental defects predominantly in CNS and heart (data not shown) demonstrates that ASPP2 performs an important, nonredundant, tissue-specific developmental function. Nevertheless, functional redundancy between ASPP1 and ASPP2 may explain the observation that other tissues did not exhibit gross abnormalities in ASPP2-null mice.

\section{ASPP2 heterozygosity confers tumor susceptibility}

Since all ASPP2-null mice died before 1 mo of age, the potential role of ASPP2 as a tumor suppressor was studied by comparing the susceptibility of ASPP2 wild-type and heterozygous mice to develop spontaneous tumors over a 3 -yr period. The number of tumor-bearing mice was significantly higher in ASPP2 heterozygotes than in wild-type mice $(p=0.02)$ (Fig. 2A). B-cell lymphomas $(40 \%)$, sarcomas (two rhabdomyosarcomas and one angiosarcoma, $15 \%)$, and squamous cell carcinoma $(5 \%)$ were observed in ASPP2-heterozygous mice (Fig. 2B,C). Wild-type mice, however, developed only a small percentage of B-cell lymphomas (22\%) (Fig. 2B). The origin of lymphomas was determined by the expression of specific markers of $\mathrm{B}$ and $\mathrm{T}$ cells (B220 and CD3, respectively) (data not shown). Extranodal (lung, kidney, liver, adrenal gland, salivary gland, and liver) lymphomas were sometimes found at lower frequency and independently of ASPP2 genotype. These findings suggest that reduced expression of ASPP2, caused by the loss of only one allele, is sufficient to confer tumor susceptibility in aged mice. The fact that ASPP2-heterozygous mice are prone to develop tumors provides first genetic evidence that ASPP2 is a tumor suppressor.

\section{ASPP2 heterozygosity accelerates tumor development in response to $\gamma$-irradiation}

Ionizing radiation is a well-known tumorigenic agent, which preferentially induces thymic lymphomas in mice. The tumor suppressor p53 plays a critical role in protecting mice from developing radiation-induced thymic lymphomas through its ability to trigger apoptosis in response to radiation (Kemp et al. 1994). We previously showed that ASPP2 activates p53-dependent apoptosis in human tumor cell lines (Samuels-Lev et al. 2001); hence, ASPP2-heterozygous mice may have an increased susceptibility to $\gamma$-irradiation-induced tumori-
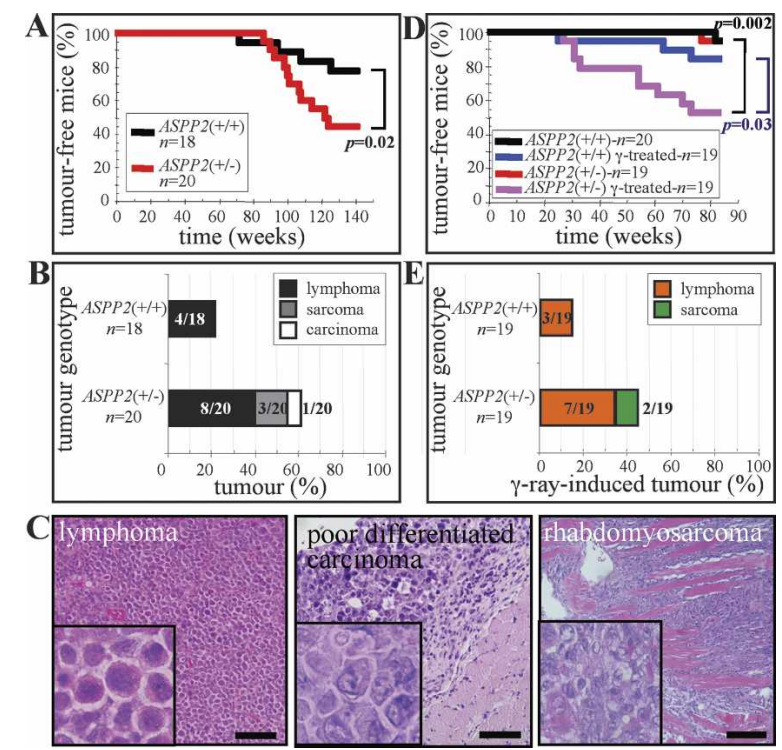

Figure 2. Heterozygosity of ASPP2 predisposes the mice to develop tumors. Each genotype group consisted of nine to 10 males and nine to 10 females. Over a 140 -wk period, some of the wild-type and heterozygous aging ASPP2 mice developed spontaneous tumors. A shows the onset and $B$ shows the percentage and spectrum of tumor types spontaneously developed in wild-type and heterozygous ASPP2 mice. Examples of histologically diagnosed thymic lymphoma, rhabdomyosarcoma, and poorly differentiated carcinoma derived from ASPP2-heterozygous mice are shown in C. Bar, $100 \mu \mathrm{m}$. $D$ shows the onset and $E$ shows the percentage and spectrum of tumor types developed in wild-type and heterozygous ASPP2 mice in response to 4 Gy $\gamma$-irradiation at P21. 
genesis. To test this, either heterozygous or wild-type mice for ASPP2 were exposed to a single dose of 4 Gy whole-body $\gamma$-irradiation at 3 wk of age. A similar group of genetic background-matched wild-type and ASPP2heterozygous mice was left untreated. Both groups of mice were observed concurrently to determine the spontaneous rate of tumor development. At $85 \mathrm{wk}$ of age, the number of tumors found in $\gamma$-irradiated $(3 / 19)$ versus untreated $(1 / 20)$ wild-type mice was similar $(p=0.25)$ (Fig. $2 \mathrm{D})$. In contrast, the number of tumor-bearing ASPP2 heterozygotes was significantly higher in $\gamma$-irradiated $(9 /$ 19) than in untreated $(1 / 19)$ mice $(p=0.002)$ (Fig. 2D). Additionally, $\gamma$-irradiated ASPP2-heterozygous mice developed significantly more tumors than $\gamma$-irradiated wild-type mice (3/19, $p=0.03$ ) (Fig. 2D). T-cell lymphomas in thymus, lung, liver, and salivary gland were found for both genotypes (17\% for ASPP2 wild-type and $35 \%$ for $A S P P 2^{+/-}$mice). Interestingly, rhabdomyosarcomas were only found in ASPP2-heterozygous mice (Fig. $2 \mathrm{E})$. These results suggest that the loss of one ASPP2 allele is sufficient to increase the susceptibility and the onset of tumor development in response to $\gamma$-irradiation. The accelerated onset of tumor development by $\gamma$-radiation was seen in $p 53^{+/-}$mice (Kemp et al. 1994). Hence, the data shown here is in agreement with the hypothesis that reduced ASPP2 expression might decrease p53 activity.

\section{ASPP2- and p53-null mutations are synthetic lethal}

To investigate the role of ASPP2 as an activator of p53 in vivo, we intercrossed double heterozygotes $A S P P 2^{+/-} p 53^{+/-}$. Among the 327 pups examined (P5-P20), mice with all combinations of ASPP2 and p53 deletions were obtained (Fig. 3A-C), with the exception of double homozygotes ASPP2 $2^{-1-} p 53^{-1-}$, which were not observed postnatally (Fig. 3C). If the expected number of pups born with each genotype was set as $100 \%$ based on Mendelian segregation, the number of ASPP2-null pups born was $70 \%$ of the expected ratio in the wild-type p53 background (Fig. $3 \mathrm{~A}, \mathrm{D})$ and decreased to $20 \%$ in the heterozygous p53 background (Fig. 3B,D) and to $0 \%$ in p53-null background (Fig. 3C,D). Thus, the number of ASPP2-null pups detected depends on $p 53$ gene dosage.

To determine at which stage the double-ASPP2/p53null embryos died during the development, we performed a timed-mating study from E11.5 to E17.5. We found no doubly homozygous embryos $(n=158$ embryos examined), far below the expected Mendelian frequency of $6.25 \%$ (Fig. 3E). Hence, all ASPP2/p53 double-homozygous-null embryos appear to die in the first half of gestation. This phenotype is in contrast with the observations that both ASPP2-null and the p53-null embryos are still viable E17.5. Altogether, these results illustrate that ASPP2- and p53-null mutations are synthetic lethal.

Genetically synthetic lethal phenotypes have been used to identify genes with an overlapping function (Griffin et al. 2001; Gurley and Kemp 2001). It refers to an unexpected or unique phenotype in a double mutant, not present in either of the original single mutants or not predictable as a simple "additive" phenotype. A "synthetic lethal" means that a strain carrying both mutations is nonviable, while parent strains with each single mutation are fully viable. This is in complete agreement with existing biochemical data that ASPP2 interacts with p53 in vitro and in vivo. The results shown here

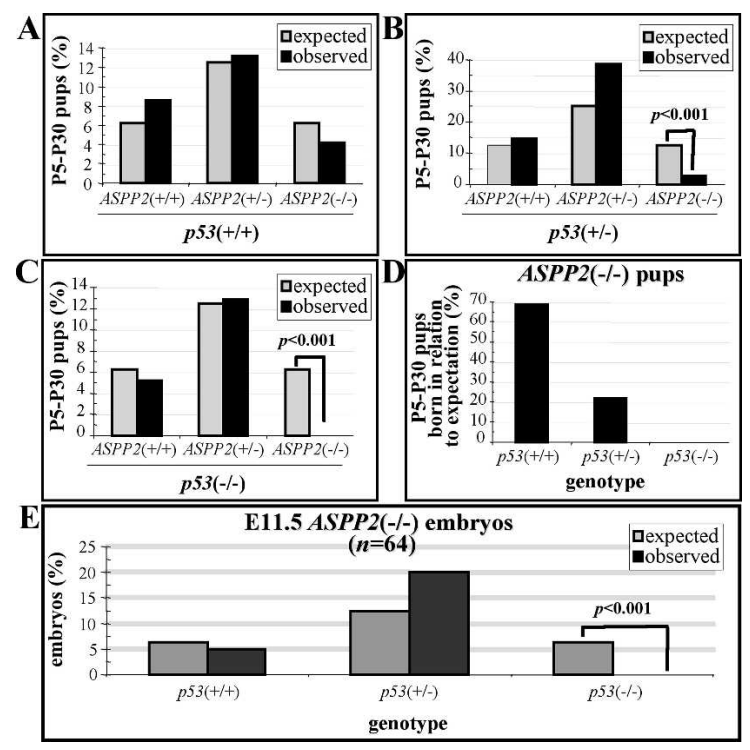

Figure 3. Overlapping functions of $p 53$ and ASPP2 in mouse development. $A-D$ show that the postnatal lethality of ASPP2-null pups depends on $p 53$ gene dosage and that deletion of both ASPP2 and p53 is synthetic lethal. In a cohort of 327 pups born from

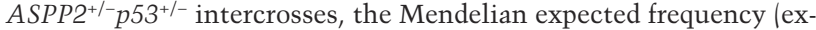
pected) and the observed frequency (observed) were compared in mice born with different ASPP2 genotypes in p53 wild-type $(A)$, heterozygous $(B)$, or null backgrounds $(C)$. If the Mendelian expected frequency is set as $100 \%$, the percentage of ASPP2-null pups born with different status is shown in $D$. E shows the Mendelian expected frequency (expected) and the observed frequency (observed) of E11.5 ASPP2-null embryos in different p53 backgrounds.

demonstrated for the first time that ASPP2 and p53 are genetically linked and that they share overlapping functions that are essential in mouse development.

Cooperation between ASPP2 and $\mathrm{p} 53$ in tumor suppression

To determine whether the loss of ASPP2 cooperates with p53 in tumorigenesis, we monitored the survival and tumor incidence in compound mutant mice with varying gene dosages of ASPP2 and p53 for a year and a half. None of the wild-type mice developed tumors at this stage of study. The percentage of tumors developed in ASPP2 ${ }^{+/-} p 53^{+/+}$and $A S P P 2^{+/+} \mathrm{p} 53^{+/-}$mice were $14 \%$ and $7 \%$, respectively (Fig. 4A,B). Interestingly, $\sim 26 \%$ of ASPP2 and p53 double-heterozygous mice developed tumors over the 72 -wk period. The tumor onset between ASPP2 ${ }^{+/+} p 53^{+/-}$and $A S P P 2^{+/-} p 53^{+/-}$mice is significantly different $(p<0.01)$ (Fig. 4A). Whereas, in the absence of p53, tumor latency was unaffected by the expression level of ASPP2, since ASPP2 ${ }^{+/+} p 53^{-/-}$and $A S P P 2^{+1}$ $-p 53^{-/-}$mice developed tumors with similar kinetics $(62 \%$ vs. $71 \%, p=0.9)$ (Fig. 4A). Pathological examination revealed that the most frequently observed tumors were lymphomas (Fig. 4B), and they were observed in both ASPP2 wild-type and heterozygous backgrounds. They were mainly detected in the thymus, but extranodular lymphomas were also found in the lung, liver, salivary gland, stomach, and kidney regardless of the genotype. Interestingly, sarcomas were again only found in ASPP2-heterozygous mice regardless of the $p 53$ status. They were located on the forelimbs, back, abdomen, and 

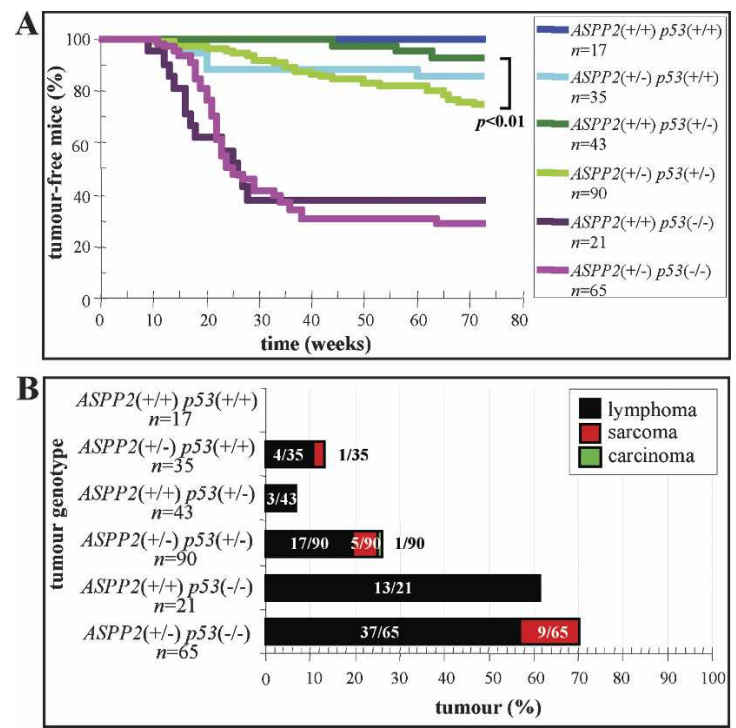

Figure 4. Cooperation of $p 53$ and ASPP2 in tumor suppression. A shows the onset and $B$ shows the percentage and spectrum of tumor types spontaneously developed in compound ASPP2/p53 mutant mice. $(n)$ Number of mice examined for each indicated genotype.

chest wall. These results clearly demonstrate that reduced ASPP2 expression confers tumor susceptibility, and this effect of ASPP2 becomes more pronounced in a p53-heterozygous background. But the onset of lymphomas in p53-null background is the same regardless of the number of ASPP2 copies.

Together with the previously established biochemical interaction of ASPP2 and p53 (Samuels-Lev et al. 2001), these data suggested that, genetically, ASPP2 is likely to function upstream of p53. Cooperating with p53 to suppress tumor growth is the underlying molecular explanation of why all ASPP2 contact residues in p53 (178, 181,243 , and 247) are mutated in human cancers with a relatively high frequency, even though none of them contact DNA. Importantly, the most frequently mutated p53 sites (273 and 248) contact both DNA and ASPP2 (Gorina and Pavletich 1996; Egloff et al. 1997). The ability of ASPP2 to cooperate with p53 to suppress tumor growth also provides a molecular explanation as to why the frequency to down-regulate ASPP2 expression tends to be higher in tumor cells expressing wild-type than mutant p53 (Samuels-Lev et al. 2001; Liu et al. 2005).

p53 is required for ASPP2 to suppress the development of lymphomas but not sarcomas

Further examination revealed that the onset of lymphoma development was accelerated in $A S P P 2^{+/-} p 53^{+/-}$ mice in comparison to that observed in $\mathrm{ASPP}^{+/+} \mathrm{p} 53^{+/-}$ and $A S P P 2^{+/-} p 53^{+/+}$mice. At $42 \mathrm{wk}$, there is a significant difference of the lymphoma onset between $A S P P 2^{+/+} p 53^{+/-}$ and $A S P P 2^{+/-} p 53^{+/-}$mice $(p=0.048)$ (Fig. 5A). Interestingly, however, at $72 \mathrm{wk}$, the difference in lymphoma onset among these two groups was no longer significant $(p=0.07)$ (Fig. 5A). Both ASPP2 ${ }^{+/+} p 53^{-/-}$and $A S P P 2^{+/}$ $-p 53^{-/-}$mice developed lymphomas with a very similar kinetics $(p=0.3)$ (Fig. 5A). The spectrum of subtypes of lymphomas ( $\mathrm{T}$ or $\mathrm{B}$ cell) developed in p53-null background is similar regardless of the number of ASPP2 copies (Fig. 5B).
These results illustrate that reduced expression level of ASPP2 weakens the tumor suppression function of $\mathrm{p} 53$. As a result, ASPP2 heterozygosity reduces the latency of tumor development. Consistent with this, the lymphoma onset between $A S P P 2^{+/+} p 53^{+/-}$and $A S P P 2^{+/-} p 53^{+/-}$mice was significantly different at $42 \mathrm{wk}$, but less so at a later stage of the study, even though the total number of tumors developed in $A S P P 2^{+/-} p 53^{+/-}$mice is significantly higher than that in $A S P P 2^{+/+} p 53^{+/-}$mice $(p<0.01$ ) (Fig. 4A). This is largely caused by accelerated sarcoma development in ASPP2-heterozygous mice, and this action of ASPP2 is largely independent of p53 (Fig. 4; data not shown). It remains unknown how ASPP2 suppresses sarcoma development. Nevertheless, as the latency and the spectrum of lymphomas developed in p53-null mice were not affected by the expression level of ASPP2, the results further indicated that ASPP2 and p53 operate on a largely overlapping pathway to suppress lymphoma development. This is consistent with the notion that ASPP2 is an upstream regulator of p53.

\section{ASPP2 is a haploinsufficient tumor suppressor gene}

Loss of heterozygosity (LOH) of the ASPP2 gene was examined in lymphomas and sarcomas derived from ASPP2-heterozygous mice. LOH of $p 53$ was also determined in tumors carrying a single copy of $p 53$. Wild-type ASPP2 allele is present in all of the tumors examined $(n=15)$. In contrast, $\mathrm{LOH}$ of $p 53$ was detected in two of the three examined tumors derived from ASPP2 $2^{+/} p 53^{+/-}$ mice (data not shown). This suggests that ASPP2 is likely to be a new haploinsufficient tumor suppressor.

The mutation or loss of one allele of haploinsufficient tumor suppressor genes is sufficient to exert a cellular phenotype that leads to tumorigenesis without inactivation of the remaining wild-type allele (Santarosa and

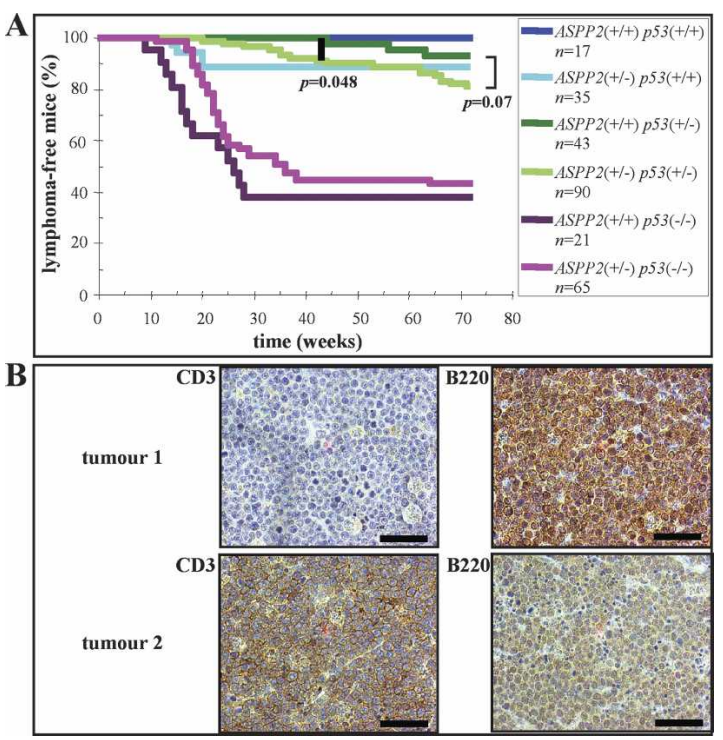

Figure 5. p53 is required for ASPP2 to suppress lymphoma development. $A$ shows the onset of spontaneous lymphomas developed in compound ASPP2/p53 mutant mice. Immunohistochemistry staining was performed to diagnose the lymphomas origin by detecting the expression of T- and B-cell surface markers, CD3 and B220, respectively. An example of such staining is shown in B. Bar, $100 \mu \mathrm{m}$. 
Ashworth 2004). Tumors influenced by haploinsufficiency usually have a later age of onset when compared with those caused by inactivation of the second allele, as observed with ASPP2-heterozygous mice, which tend to develop observable spontaneous tumors at $\sim 3 \mathrm{yr}$ of age (Fig. 2A). Moreover, deletion and mutation of ASPP2 has not yet been detected in a number of human tumor cell lines and tumor samples tested so far (data not shown). Instead, frequent down-regulation of ASPP2 expression was observed in many human tumor cell lines and tumor samples (Samuels-Lev et al. 2001; Liu et al. 2004). Moreover, reduced ASPP2 expression is often caused by methylation (Liu et al. 2005; X. Lu, unpubl.). Importantly, low levels of ASPP2 expression correlate with a poor clinical outcome in human diffuse large B-cell lymphomas (Lossos et al. 2002). Hence, increasing the ASPP2 expression provides a novel molecular approach to allow the restoration of the apoptotic function of p53 in human cancer cells. Molecules that increase the expression level of ASPP2 would undoubtly enhance the efficacy of cancer treatments.

\section{Materials and methods}

Targeted disruption of the ASPP2 gene

ASPP2 gene targeting was performed by InGenious Targeting Laboratory Inc. Briefly, the mouse ASPP2 gene was cloned from the 129SVJ phage genomic library and exons were mapped. A targeting vector was constructed using, as the short arm, a 1.3-kb PCR product from primers X23 to $\mathrm{X} 22$ and, as the long arm, a fragment from EcoRV to the end of the ASPP2 $\lambda$ genomic clone. Primer X23 is located inside intron 2, 130 bp downstream of exon 3, with a sequence of 5 '-TGGGATGAAGG GAAGCTAGGAC-3'. Primer X22 is also located inside intron 3, a further $1.4 \mathrm{~kb}$ downstream of exon 3 with a sequence of $5^{\prime}$-CTTTCT GTCCTATATCAACTC-3'. In this knockout strategy, exon 3 and $2 \mathrm{~kb}$ of upstream sequence were replaced by the neo gene cassette. Ten micrograms of targeting plasmid DNA were linearized with NotI and electroporated into IT2 ES cells. After selection in G418, surviving colonies were expanded, and PCR analysis was performed to identify clones that had undergone homologous recombination. Correctly targeted ES cell clones were microinjected into $\mathrm{C} 57 \mathrm{BL} / 6 \mathrm{~J}$ host blastocysts to generate chimeric mice, which gave germline transmission of the disrupted ASPP2 gene. Genotyping was performed by PCR using primers $\mathrm{a}, \mathrm{b}$, and c. Primer a is located inside intron 2400 bp upstream of exon 3, with a sequence of 5'-CCTCTCACAAAAGGAAATAACCTG-3'. Primer b is located in intron $4545 \mathrm{bp}$ downstream of exon 3 with a sequence of 5'-AGGAAAACCACCACCTTCAC-3'. Primer c is located in the neo gene cassette and has a sequence of $5^{\prime}$-TACCCGCTTCCATTGCTCAG$3^{\prime}$. In the presence of the knockout allele, primers b and c amplify a PCR product of $1.1 \mathrm{~kb}$. In the presence of the wild-type allele, primers $\mathrm{a}$ and $\mathrm{b}$ amplify a fragment of $0.9 \mathrm{~kb}$.

\section{Mouse colonies}

p53-heterozygous mice (on a $129 \mathrm{SvJxC57BL/6}$ background) were obtained from the Jackson Laboratories and were crossed once with C57BL/ $6 \mathrm{~J}$ to generate the p53-heterozygous (C57BL/6Jx129SvJ) colony used in the study. ASPP2 $2^{+/-}$mice (also on a C57BL/6Jx129SvJ background) were

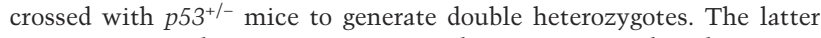
were intercrossed to generate compound genotypes. $p 53$ knockout mice were genotyped using the following primers: $5^{\prime}$-CCCGAGTATCTG GAAGACAG-3' (exon 6), 5'-ATAGGTCGGCGGTTCAT-3' (exon 7), and 5'-GGACCGCTATCAGGACATA-3' (neomycin resistance gene).

\section{RT-PCR for ASPP2}

Total RNA was isolated from brain samples using Trizol according to the manufacturer's instructions (Invitrogen). Three micrograms of total RNA was reverse-transcribed using the SuperScript first-strand synthesis system for RT-PCR (Invitrogen), and the resulting templates were subjected to PCR with ASPP2-specific primers located in exon 2 (5'-GCCACC TGGGCAGACAAGACAGGTAA-3') and exon 4 (5'-ACCCCATTCTC
CTTCCTCCGATGC-3'). The amplification of exons 2, 3, 4, and 2, 4 resulted in products of 368 and $268 \mathrm{bp}$, respectively.

Western blot analysis of ASPP2

A sequence encoding amino acids 59-383 of ASPP2 was inserted in-frame with the His-coding sequence in $\mathrm{pCR} / \mathrm{T} 7$ (Invitrogen). The resultant fusion protein was expressed in bacteria and purified. Antibodies were generated in rabbits and the serum was named S32. Protein extracts were prepared from $A S P P 2^{+/+}, A S P P 2^{+/-}$, and $A S P P 2^{-/-}$E13.5 brains, and Western blot analysis was performed using the $\mathrm{S} 32$ antibody.

\section{Histology and immunohistochemistry}

Pups' organs and tumors were fixed in 10\% buffered formalin overnight and then dehydrated in an ethanol series, cleared in xylene, and embed-

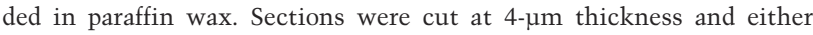
stained with hematoxylin and eosin or processed for immunostaining. Rehydrated paraffin-embedded sections were microwaved in $10 \mathrm{mM}$ sodium citrate buffer ( $\mathrm{pH} 6)$, incubated in $3 \%$ hydrogen peroxide in methanol, washed in PBS, and incubated overnight with the primary antibody at $4^{\circ} \mathrm{C}$, followed by biotinylated secondary antibody for $1 \mathrm{~h}$ at room temperature. Staining was visualized using the peroxide substrate solution DAB (diaminobenzidine, Vector). Primary antibodies were rabbit anti-CD3 (1:200, Abcam) and mouse anti-B220 (1:200, Pharmingen).

\section{Tumor analysis}

Mice that developed visible tumors or showed signs of ill health were killed and subjected to complete necropsy with careful examination for tumors, tissues with possible neoplasm, and other abnormalities. Tumors and samples from brain, eyes, salivary gland, thymus, heart, lung, liver, spleen, stomach, bowel, kidney, uterus, and testis were fixed in $10 \%$ buffered formalin, and processed for paraffin histology. Four-micrometer-thick sections were stained with hematoxylin and eosin.

\section{Statistical analysis}

Comparisons between genotype frequencies and between the types of tumors developed by mice of different genotypes were performed using the $\chi^{2}$ test. The log-rank test was used to determine the statistical significance of differences in tumor-free survival of different genotypes.

\section{Acknowledgments}

We thank Steve Pullen, Chris Staines, Glynis Musselwhite, and Anne Keeley for advice, technical support, and discussions. This work was funded by the Ludwig Institute for Cancer Research (to X.L.) and Association for International Cancer Research (to X.L.). J.S. is funded by Mackay Memorial Hospital, Taipei, Taiwan.

\section{References}

Bergamaschi, D., Samuels, Y., O'Neil, N.J., Trigiante, G., Crook, T., Hsieh, J.K., O'Connor, D.J., Zhong, S., Campargue, I., Tomlinson, M.L., et al. 2003. iASPP oncoprotein is a key inhibitor of p53 conserved from worm to human. Nat. Genet. 33: 162-167.

Bergamaschi, D., Samuels, Y., Jin, B., Duraisingham, S., Crook, T., and Lu, X. 2004. ASPP1 and ASPP2: Common activators of p53 family members. Mol. Cell. Biol. 24: 1341-1350.

Chen, Y., Liu, W., Naumovski, L., and Neve, R.L. 2003. ASPP2 inhibits APP-BP1-mediated NEDD8 conjugation to cullin-1 and decreases APP-BP1-induced cell proliferation and neuronal apoptosis. J. Neurochem. 85: 801-809.

Egloff, M.P., Johnson, D.F., Moorhead, G., Cohen, P.T., Cohen, P., and Barford, D. 1997. Structural basis for the recognition of regulatory subunits by the catalytic subunit of protein phosphatase 1. EMBO $\mathrm{J}$. 16: $1876-1887$.

Espanel, X. and Sudol, M. 2001. Yes-associated protein and p53-binding protein-2 interact through their WW and SH3 domains. J. Biol. Chem. 276: $14514-14523$.

Gorina, S. and Pavletich, N.P. 1996. Structure of the p53 tumor suppressor bound to the ankyrin and SH3 domains of 53BP2. Science 274: 1001-1005.

Griffin, C.T., Srinivasan, Y., Zheng, Y.W., Huang, W., and Coughlin, S.R. 2001. A role for thrombin receptor signaling in endothelial cells dur- 
ing embryonic development. Science 293: 1666-1670.

Gurley, K.E. and Kemp, C.J. 2001. Synthetic lethality between mutation in Atm and DNA-PK(cs) during murine embryogenesis. Curr. Biol. 11: 191-194.

Helps, N.R., Barker, H.M., Elledge, S.J., and Cohen, P.T. 1995. Protein phosphatase 1 interacts with p53BP2, a protein which binds to the tumour suppressor p53. FEBS Lett. 377: 295-300.

Iwabuchi, K., Bartel, P.L., Li, B., Marraccino, R., and Fields, S. 1994. Two cellular proteins that bind to wild-type but not mutant p53. Proc. Nat1. Acad. Sci. 91: 6098-6102.

Kemp, C.J., Wheldon, T., and Balmain, A. 1994. p53-deficient mice are extremely susceptible to radiation-induced tumorigenesis. Nat. Genet. 8: 66-69.

Liu, Z.J., Zhang, Y., Zhang, X.B., and Yang, X. 2004. Abnormal mRNA expression of ASPP members in leukemia cell lines. Leukemia 18: 880.

Liu, Z.J., Lu, X., Zhang, Y., Zhong, S., Gu, S.Z., Zhang, X.B., Yang, X., and Xin, H.M. 2005. Downregulated mRNA expression of ASPP and the hypermethylation of the $5^{\prime}$-untranslated region in cancer cell lines retaining wild-type p53. FEBS Lett. 579: 1587-1590.

Lossos, I.S., Natkunam, Y., Levy, R., and Lopez, C.D. 2002. Apoptosis stimulating protein of p53 (ASPP2) expression differs in diffuse large B-cell and follicular center lymphoma: Correlation with clinical outcome. Leuk. Lymphoma 43: 2309-2317.

Nakagawa, H., Koyama, K., Murata, Y., Morito, M., Akiyama, T., and Nakamura, Y. 2000. APCL, a central nervous system-specific homologue of adenomatous polyposis coli tumor suppressor, binds to p53binding protein 2 and translocates it to the perinucleus. Cancer Res. 60: 101-105.

Naumovski, L. and Cleary, M.L. 1996. The p53-binding protein 53BP2 also interacts with $\mathrm{Bc} 12$ and impedes cell cycle progression at G2/M. Mol. Cell. Biol. 16: 3884-3892.

Samuels-Lev, Y., O'Connor, D.J., Bergamaschi, D., Trigiante, G., Hsieh, J.K., Zhong, S., Campargue, I., Naumovski, L., Crook, T., and Lu, X. 2001. ASPP proteins specifically stimulate the apoptotic function of p53. Mol. Cell 8: 781-794.

Santarosa, M. and Ashworth, A. 2004. Haploinsufficiency for tumour suppressor genes: When you don't need to go all the way. Biochim. Biophys. Acta 1654: 105-122.

Slee, E.A. and Lu, X. 2003. The ASPP family: Deciding between life and death after DNA damage. Toxicol. Lett. 139: 81-87.

Yang, J.P., Hori, M., Takahashi, N., Kawabe, T., Kato, H., and Okamoto, T. 1999. NF-кB subunit $\mathrm{p} 65$ binds to 53BP2 and inhibits cell death induced by 53BP2. Oncogene 18: 5177-5186. 


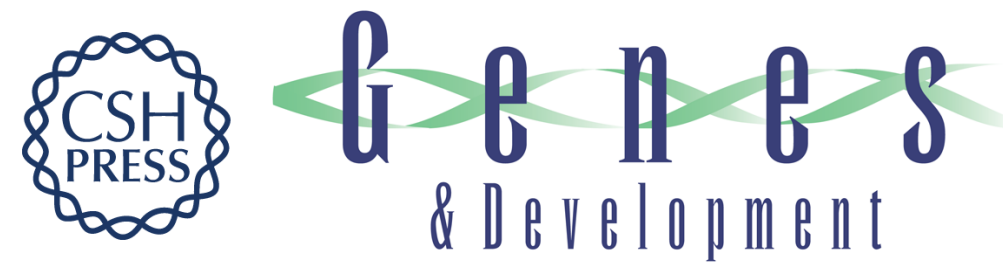

\section{ASPP2 is a haploinsufficient tumor suppressor that cooperates with p53 to suppress tumor growth}

Virginie Vives, Jian Su, Shan Zhong, et al.

Genes Dev. 2006, 20:

Access the most recent version at doi:10.1101/gad.374006

References This article cites 20 articles, 8 of which can be accessed free at: http://genesdev.cshlp.org/content/20/10/1262.full.html\#ref-list-1

License

Email Alerting

Receive free email alerts when new articles cite this article - sign up in the box at the top Service right corner of the article or click here.

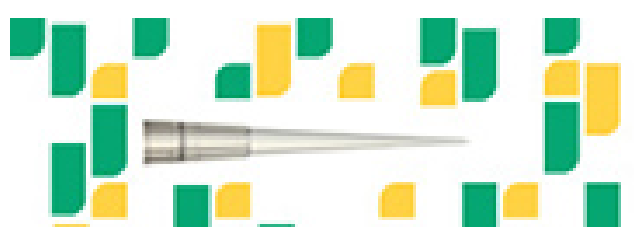

Focused on your science. 